\title{
Polystyrene/Montmorillonite Nanocomposites: Study of the Morphology and Effects of Sonication Time on Thermal Stability
}

\author{
Mashael Alshabanat, ${ }^{1}$ Amal Al-Arrash, ${ }^{2}$ and Waffa Mekhamer ${ }^{2}$ \\ ${ }^{1}$ Department of Chemistry, Science College, Princess Nora Bint Abdu Rahman University, Riyadh 1167, Saudi Arabia \\ ${ }^{2}$ Department of Chemistry, Science College, King Saud University, Riyadh 1145, Saudi Arabia
}

Correspondence should be addressed to Mashael Alshabanat; ma.naif@hotmail.com

Received 4 November 2012; Revised 4 January 2013; Accepted 5 January 2013

Academic Editor: Fathallah Karimzadeh

Copyright ( 2013 Mashael Alshabanat et al. This is an open access article distributed under the Creative Commons Attribution License, which permits unrestricted use, distribution, and reproduction in any medium, provided the original work is properly cited.

Polymer nanocomposites of polystyrene matrix containing 10\% wt of organo-montmorillonite (organo-MMT) were prepared using the solution method with sonication times of $0.5,1,1.5$, and 2 hours. Cetyltrimethylammonium bromide (CTAB) is used to modify the montmorillonite clay after saturating its surface with $\mathrm{Na}^{+}$ions. Fourier transform infrared spectroscopy (FTIR), X-ray diffraction (XRD), scanning electron microscopy (SEM), and transmission electron microscopy (TEM) were used to characterize the montmorillonite before and after modification by CTAB. The prepared nanocomposites were characterized using the same analysis methods. These results confirm the intercalation of PS in the interlamellar spaces of organo-MMT with a very small quantity of exfoliation of the silicate layers within the PS matrix of all samples at all studied times of sonication. The thermal stability of the nanocomposites was measured using thermogravimetric analysis (TGA). The results show clear improvement, and the effects of sonication time are noted.

\section{Introduction}

Recently, nanocomposites, such as polymer-layered silicate nanocomposites, have become effective alternatives to conventional polymer composites in many applications. These systems may be classified according to the dispersion of clay in the polymer. In general, two types of polymer-layered clay nanocomposites can be obtained, intercalated, and exfoliated, and the latter are known as delaminated nanocomposites. Nanocomposites are defined as composites in which the dispersed particles are in the nanometer range in at least one dimension $[1,2]$. The thickness of the layer in layered clay is approximately $1 \mathrm{~nm}$, and the lateral dimensions of these layers may vary from $300 \AA$ to several microns or even larger depending on the particular silicate. The layers organize to form stacks with a regular van der Waals gap, which is called the interlayer [3]. Intercalates are obtained when a single polymer chain is located between silicate layers in a manner that increases layer spacing while attractive forces between the layers maintain regularly spaced stacks. Exfoliates are obtained when the layer spacing is sufficiently increased to overcome the interactions between the layers, which are randomly dispersed in a continuous polymer matrix [4]. The preparation method of nanocomposites can be classified into three categories according to the starting materials, and processing techniques: intercalation polymerization, which includes the intercalation of one or more suitable monomers and subsequent polymerization [5-8]; polymer intercalation by the solution method [9-11]; and polymer intercalation by the melt method [12]. However, melt intercalation is advantageous because of the absence of the need for organic solvents, which makes this method environmentally benign, and because of the ability to adopt processing techniques such as extrusion and injection molding, the solution method gives good control on the homogeneity of the constituents, which helps to understand the intercalation process and nanocomposite morphology. It also leads to a better understanding of the structure and dynamics of the intercalated polymers 
TABLE 1: Product data for PS-330.

\begin{tabular}{lccc}
\hline Property & Unit & Value & Test method \\
\hline Melt index $\left(200^{\circ} \mathrm{C}, 5 \mathrm{KG}\right)$ & $\mathrm{g} / 10 \mathrm{~min}$ & 3.0 & ASTMD-1238 \\
Density $\left(23^{\circ} \mathrm{C}\right)$ & $\mathrm{g} / \mathrm{cm}^{3}$ & 1.04 & $\mathrm{D}-792$ \\
Bulk density (Method B) & $\mathrm{g} / \mathrm{cm}^{3}$ & 0.60 & $\mathrm{D}-1895$ \\
Ultimate tensile elongation & $\%$ & 50 & ASTMD-638 \\
Ultimate tensile strength & $\mathrm{MPa}$ & 29 & $\mathrm{ASTMD}-638$ \\
Tensile modulus & $\mathrm{MPa}$ & 443 & $\mathrm{ASTMD}-638$ \\
Flexural strength & $\mathrm{MPa}$ & 2647 & ASTMD-790 \\
Flexural modulus & $\mathrm{MPa}$ & 110 & ASTMD-790 \\
Notched Izod impact strength at $23^{\circ} \mathrm{C}$ & $\mathrm{J} / \mathrm{m}$ & 99 & ASTMD-256 \\
Victa softing temperature & ${ }^{\circ} \mathrm{C}$ & 67 & ASTMD-1525 \\
Rockwell hardness $(\mathrm{L}-\mathrm{Scale})$ & - & ASTMD-785 \\
\hline
\end{tabular}

TABLE 2: The chemical composition of raw montmorillonite.

\begin{tabular}{lcccccccc}
\hline $\mathrm{SiO}_{2}$ & $\mathrm{Al}_{2} \mathrm{O}_{3}$ & $\mathrm{Fe}_{2} \mathrm{O}_{3}$ & $\mathrm{CaO}$ & $\mathrm{MgO}$ & $\mathrm{Na}_{2} \mathrm{O}$ & $\mathrm{K}_{2} \mathrm{O}$ & $\mathrm{TiO}_{2}$ & Impurities \\
\hline $70.30 \%$ & $15 \%$ & $7.75 \%$ & $1.60 \%$ & $2.30 \%$ & $1.45 \%$ & $1.20 \%$ & $0.30 \%$ & 0.10 wt. $\%$ \\
\hline
\end{tabular}

in these nanocomposites, which can provide molecular insight and lead to the design of materials with desired properties [13]. Therefore, in this work, modification of local montmorillonite clay to use as filler will be achieved by the solution method and will be used to prepare PS/organoMMT nanocomposites. The effect of sonication time, which is performed during the preparation process, on the thermal stability of the PS/organo-MMT nanocomposites will be studied. The morphology of the clay and nanocomposites will be characterized using FTIR, XRD, and TEM.

\section{Experimental}

2.1. Materials. Polystyrene (PS) was used as a matrix and was supplied by the Saudi Basic Industries Corporation (SABIC). The brand name for polystyrene is PS 330, and the details of polystyrene are given in Table 1. Raw montmorillonite (MMT) was collected from the Kholais region north of Jeddah in Saudi Arabia and ground in our laboratory. The chemical composition was determined using Xray florescence (XRF; see Table 2). The silicate surface is generally too hydrophilic to be compatible with most polymers. To render the hydrophobic clay surface, they are intercalated with organic cations such as alkylammonium salt by a cation exchange reaction. Cetyltrimethylammonium bromide (CTAB), $\mathrm{C}_{19} \mathrm{H}_{42} \mathrm{BrN}$, with a molecular weight of $364.45 \mathrm{~g} / \mathrm{mol}$ and a purity of $99 \%$, which was provided by $\mathrm{BDH} \& \mathrm{Co}$, was used as a surfactant. Toluene, $\mathrm{C}_{6} \mathrm{H}_{5} \mathrm{CH}_{3}$, which has a molecular weight of $92.14 \mathrm{~g} / \mathrm{mol}$ and a purity of 99.5\%, was provided by BDH \& Co. Sodium chloride $(\mathrm{NaCl})$, molecular weight $(58.44 \mathrm{~g} / \mathrm{mol})$.

\subsection{Preparation Methods}

2.2.1. Washing and Saturation of Montmorillonite. To prepare the MMT suspension, $31 \mathrm{~mL}$ of distilled water was added to $150 \mathrm{~g}$ of MMT and shaken for $24 \mathrm{~h}$. After, the suspension was allowed to sit for $30 \mathrm{~min}$. Distilled water was added to the separated MMT suspension, and the mixture was shaken for $48 \mathrm{~h}$. The mixture was allowed to sit for $24 \mathrm{~h}$. After, the MMT suspension was separated and saturated by shaking with $0.5 \mathrm{~mol} / \mathrm{L}$ of $\mathrm{NaCl}$ solution for $24 \mathrm{~h}$. This step was repeated five times. The sediment was washed with distilled water to remove excess salt. This was confirmed by a negative chloride ion test using $\mathrm{AgNO}_{3}$. The saturated montmorillonite was dried at $105^{\circ} \mathrm{C}$ and grounded. The montmorillonite was stored in well-stoppered bottles in desiccators over $\mathrm{CaCl}_{2}$. The samples of Na-saturated montmorillonite were labeled with Na-MMT.

2.2.2. Modification of Montmorillonite. The organo-MMT was prepared using the ion exchange method. A cetyltrimethylammonium bromide (CTAB) solution was used to modify Na-MMT using a concentration of CTAB that was twice that of the cation exchange capacity $(\mathrm{CEC}=80 \mathrm{meq} / 100 \mathrm{~g})$ of the montmorillonite. Ten grams of Na-MMT was dispersed in $1000 \mathrm{~mL}$ of deionized water, and the dispersion was vigorously stirred overnight. Approximately $7.3 \mathrm{~g}$ of CTAB was dissolved in $100 \mathrm{~mL}$ of deionized water and was slowly added to the Na-MMT suspension. The mixture was magnetically stirred for $24 \mathrm{~h}$ at room temperature [14]. Organo-MMT particles were separated by centrifugation at ambient temperature and repeatedly washed to remove excess $\mathrm{Br}^{-}$. The product was dried in a vacuum oven at $80^{\circ} \mathrm{C}$. The sample was labeled as organo-MMT.

2.2.3. PS/MMT Nanocomposites. Ten percent of the weight of organo-MMT was added to $20 \mathrm{~mL}$ of toluene. The suspension was stirred magnetically for $1 \mathrm{~h}$ and sonicated for $1 \mathrm{~h}$ before PS ( $2 \mathrm{~g}$ ) was added. The resulting mixture was magnetically stirred for $1 \mathrm{~h}$ and further sonicated for $0.5,1,1.5$, and $2 \mathrm{~h}$ to study the effect of sonication time. The mixture was cast into petri dishes. The thin films, which were obtained, were 
removed from the glass plates after $24 \mathrm{~h}$ at room temperature, and the solvent was evaporated. This procedure was performed at room temperature and ambient pressure. The nanocomposites obtained were labeled as PS/organo-MMT. A virgin polystyrene sample was prepared for comparison by mixing it with toluene using a magnetic stirrer. This sample was labeled as PS.

2.3. Characterization and Measurements. Particle size distribution measurements of $\mathrm{Na}-\mathrm{B}$ and $\mathrm{MB}$ suspensions were performed using Malvern Zetasizer, ZS, ZEN3500 (UK). Aqueous suspensions were prepared as follows. $0.1 \mathrm{~g}$ of clay sample was dispersed in distilled water. The suspension was kept in ultrasonic for 5 minutes in order to break powders' agglomerates resulting fine, colloidal particles dispersed in water. The structures of Na-MMT, organo-MMT powder, and the prepared nanocomposite films were investigated using Fourier-transform infrared (FTIR) spectroscopy (FIIR Spectrometer 1000, Perkin Elmer). The structure of nanocomposites were monitored using X-ray diffraction (XRD), RIGAKU Ultima-IV diffractometer (Japan), and intensity data were collected in the $2 \theta$ range of $0^{\circ}-25^{\circ}$ at a step of $0.02^{\circ}$ and a $2 \mathrm{~s}$ count time using $\mathrm{Cu}-\mathrm{K} \alpha$ radiation. The surface morphology of the prepared organoclays was examined by using a JEOL JSM-6360LV scanning electron microscope. Small amounts of the dried powders (approximately $0.01 \mathrm{~g}$ ) were placed on sticky carbon tape on standard Al mounts, then sputter coated with a thin conductive layer of gold.

Transmission electron microscopy (TEM) images were recorded to investigate the morphology and inner structure of the PS/organo-MMT nanocomposite samples. TEM images were recorded on a JEOL JSM-6060LV transmission electron microscope. TEM is at an accelerating voltage of $100 \mathrm{kV}$. For TEM observation, small drops of dilute suspensions of $0.1 \mathrm{~g}$ of the clays ( $\mathrm{Na}-\mathrm{B}$ and $\mathrm{MB}$ ) in $5 \mathrm{~cm}^{3}$ of doubly distilled water were placed on $\mathrm{Cu}$ mesh grids which had been coated with a thin carbon film. The grids were air-dried then briefly placed in a $60^{\circ} \mathrm{C}$ oven to ensure complete drying prior to insertion into the instrument. The samples of nanocomposites film were microtomed at room temperature with a diamond knife using a Reichert utramicrotome in order to obtain $80 \mathrm{~nm}$ thick sections. Microtomed sections were transferred from water onto 200 mesh copper grids and used without staining. Thermodegradation of the PS/organo-MMT nanocomposites were determined using thermo gravimetric analysis (TGA) with a Perkin-Elmer analyzer. Thermograms, using approximately $10 \mathrm{mg}$ of sample, were recorded from $25^{\circ} \mathrm{C}$ to $800^{\circ} \mathrm{C}$ at a heating rate of $10^{\circ} \mathrm{C} \mathrm{min}^{-1}$ under nitrogen flow.

\section{Results and Discussion}

3.1. Size Distribution of Montmorillonite Suspensions. Particle size distribution analysis (PSD) is a measurement designed to determine information about the size and range of a set of particles in a representative material. Particle size was determined using dynamic light scattering measurements. Figures 1 and 2 show the size distribution histogram of Na-MMT and organo-MMT suspensions. The Zetasizer software uses algorithms to extract the decay rates
TABLE 3: The particle size distribution of Na-MMT and organoMMT.

\begin{tabular}{lcccc}
\hline Samples & \multicolumn{3}{c}{ Na-MMT } & Organo-MMT \\
\hline Particle size $(\mathrm{nm})$ & 749.5 & 105.4 & 4263 & 1298 \\
Intensity \% & 88.2 & 6.4 & 5.4 & 100 \\
\hline
\end{tabular}

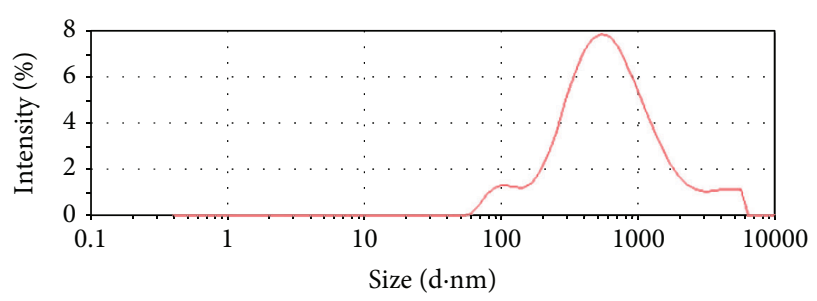

FIGURE 1: The particle size distributions of the Na-MMT suspension.



Figure 2: The particle size distributions of the organo-MMT suspension.

for a number of size classes to produce a size distribution. The $X$-axis shows a distribution of size classes, whereas the $Y$-axis shows the relative intensity of the scattered light. The particle size distribution of the Na-MMT suspension had a wide distribution (three peaks); therefore, its distribution is very heterogeneous. The particle size distribution for Na-MMT was $88.2 \%$ of the particles were $748.5 \mathrm{~nm}, 6.4 \%$ were $105.4 \mathrm{~nm}$, and $5.4 \%$ were $426.3 \mathrm{~nm}$. The organo-MMT suspension shows one peak with a narrow size distribution in the histogram, which may be related to one type of size distribution. This result revealed the particle size of organo-MMT to be highly monodispersed, indicating that they have the same size and shape with an average diameter of $1298 \mathrm{~nm}$, which was obtained with $100 \%$ intensity. The increase in the size was attributed to an aggregation of particles because of an interaction of the positively charged CTAB molecules with the negatively charged clay particles. The average size and intensity are presented in Table 3.

\subsection{Infrared Absorption Spectra}

3.2.1. Infrared Absorption Spectra of Montmorillonite. The infrared absorption spectra (FT-IR) of Na-MMT and organoMMT were recorded in the region from $400-4000 \mathrm{~cm}^{-1}$. The full FT-IR spectra of the samples are presented in Figure 3. For the Na-MMT spectrum, the absorption band at $3624 \mathrm{~cm}^{-1}$ is because of the stretching vibrations of structural $\mathrm{OH}$ groups coordinated to $\mathrm{Al}-\mathrm{Al}$ pairs, the complex broadband at approximately $1032 \mathrm{~cm}^{-1}$ corresponds to $\mathrm{Si}-\mathrm{O}$ 
TABLE 4: The infrared spectra absorption data of Na-MMT and organo-MMT.

\begin{tabular}{lccc}
\hline Assignments & Na-MMT & Organo-MMT & Shift $\left(\mathrm{cm}^{-1}\right)$ \\
\hline OH stretching of structural hydroxyl groups (Al-OH) & 3624 & 3626 & 3430 \\
OH stretching of water & 3447 & 2922 & 2 \\
Asymmetric stretching of the $\mathrm{CH}_{2}$ (in CTAB) & - & 2852 & -17 \\
Symmetric stretching of the $\mathrm{CH}_{2}$ (in CTAB) & - & 1655 & - \\
OH bending of water & 1639 & 1476 \\
$\mathrm{CH}$ bending (in CTAB) & - & 1032 \\
$\mathrm{Si}-\mathrm{O}$ stretching & 1032 & 911 & - \\
$\mathrm{Si}-\mathrm{O}$ stretching of the tetrahedral silica layers & 915 & 525 & - \\
$\mathrm{Si}-\mathrm{O}$ asymmetric bending & 528 & 465 \\
Si-O asymmetric bending & 466 & -4 \\
\hline
\end{tabular}

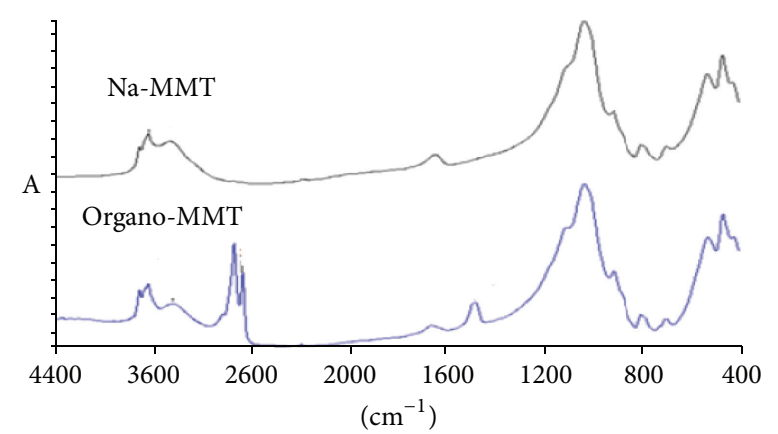

FIgURE 3: FT-IR spectra of Na-MMT and organo-MMT.

stretching, and the 528 and $467 \mathrm{~cm}^{-1}$ bands are related to Al-O-Si, Si-O-Si, and Si-O deformations [15, 16]. Adsorbed molecules of water result in a broad band at $3447 \mathrm{~cm}^{-1}$, which corresponds to the $\mathrm{H}_{2} \mathrm{O}$-stretching vibrations, and the absorption band of the $\mathrm{H}_{2} \mathrm{O}$ bending vibration was at $1639 \mathrm{~cm}^{-1}$ [17].

For the organo-MMT spectrum, there are several absorption bands that appeared at different positions. The asymmetric stretching vibration of the structural $\mathrm{OH}$ groups of $\mathrm{Na}-\mathrm{MMT}$ at $3626 \mathrm{~cm}^{-1}$, the symmetric stretching vibration of the hydroxyl groups at $3430 \mathrm{~cm}^{-1}$, and the bending inplane vibration of $\mathrm{H}-\mathrm{O}-\mathrm{H}$ at $1656 \mathrm{~cm}^{-1}$ correspond to the structural changes from hydrophilic to hydrophobic characteristics $[18,19]$. The stretching band of the $\mathrm{OH}$ groups at $3624 \mathrm{~cm}^{-1}$ (Na-MMT) shifted to $3626 \mathrm{~cm}^{-1}$ in the organo-MMT sample. This slight shift towards the smaller frequencies implies the replacement of $\mathrm{H}_{2} \mathrm{O}$ molecules from the galleries when CTAB is adsorbed. Similarly, the bending in-plane vibrations of the $\mathrm{OH}$ groups are characterized by a broad band at $1639 \mathrm{~cm}^{-1}$. This band shifted to $1656 \mathrm{~cm}^{-1}$ in the FT-IR spectrum of the organo-MMT sample, which indicates an intercalation of surfactant molecules between the silica layers in montmorillonite. The broad band observed at $3447 \mathrm{~cm}^{-1}$ corresponds to the stretching vibrations of the structural and free $\mathrm{OH}$ groups. This band shifted to $3430 \mathrm{~cm}^{-1}$ in organo-MMT. The sharp peaks that appear at

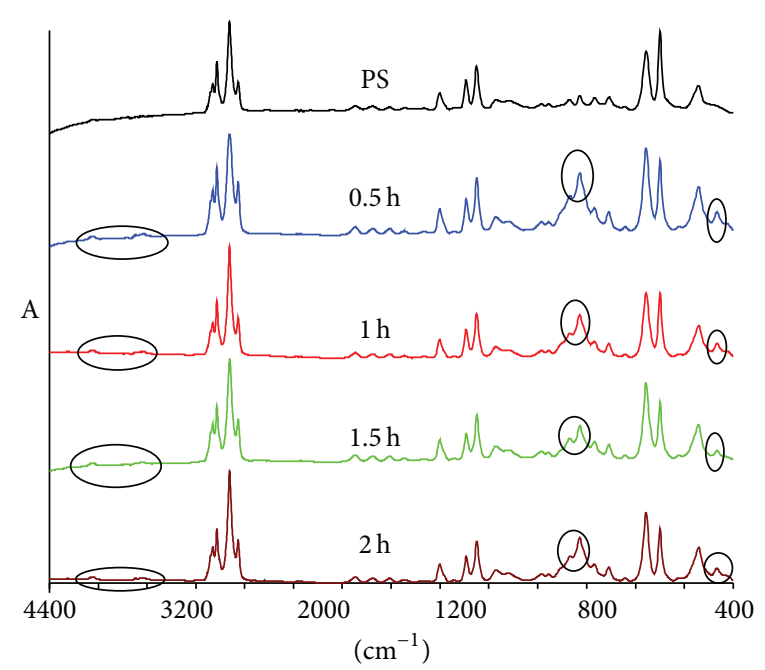

FIGURE 4: FT-IR spectra of PS and PS/MMT nanocomposites at different sonication times.

2922 and $2822 \mathrm{~cm}^{-1}$ are characteristics of CTAB, and these peaks are indicative of intermolecular attractions between adjacent alkyl chains of CTAB in Na-MMT galleries [20, 21]. A small peak appeared at $915 \mathrm{~cm}^{-1}$ and belonged to the in-plane stretching vibrations of the $\mathrm{Si}-\mathrm{O}$ bonds of the tetrahedral silica layers that shifted to $911 \mathrm{~cm}^{-1}$. All of these changes refer to the organophilic modification of Na-MMT by CTAB in the prepared sample of organo-MMT [19, 2226]. Table 4 shows the assignments of Na-MMT and organoMMT.

3.2.2. Infrared Absorption Spectra of PS and the PS Nanocomposites. The infrared absorption spectra (FT-IR) of PS and the PS/organo-MMT nanocomposites after different sonication times were recorded in the region from $400-4000 \mathrm{~cm}^{-1}$, as shown in Figure 4. The infrared spectrum of PS features bands at 3066, 3025, 2922, 2851, 1666-1945, 1491-1599, $1188-1368,1026,698-756$, and $543 \mathrm{~cm}^{-1}$, and salient absorption bands and explanations are listed in Table 5. The FTIR spectra of the PS/organo-MMT nanocomposite at all applied times of sonication clearly exhibit the characteristic 


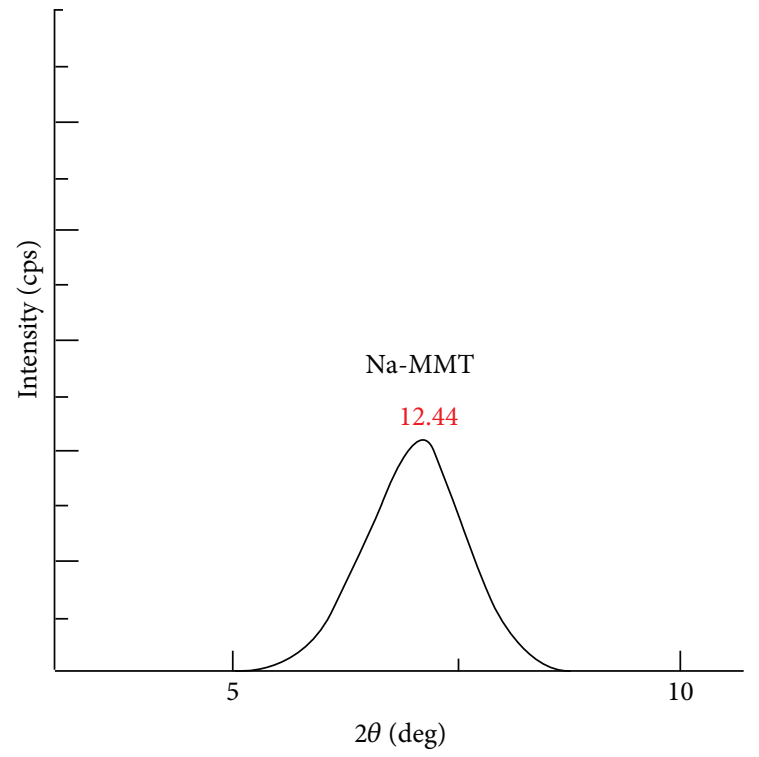

(a)



(b)

FIGURE 5: X-ray diffraction patterns of Na-MMT and organo-MMT.

TABLE 5: Positions and assignments of the IR vibration bands of PS.

\begin{tabular}{|c|c|}
\hline Wavenumber $\left(\mathrm{cm}^{-1}\right)$ & Assigment \\
\hline 3066 and 3026 & Stretching vibration of aromatic $\mathrm{C}-\mathrm{H}$ \\
\hline 2922 and 2851 & Stretching vibration of aliphatic $\mathrm{C}-\mathrm{H}$ \\
\hline $1666-1945$ & $\begin{array}{l}\text { Overton and combination bands (mono } \\
\text { substituted aromatic) }\end{array}$ \\
\hline 1491, 1449, and 1599 & Stretching vibration of aromatic $\mathrm{C}=\mathrm{C}$ \\
\hline $1188-1368$ & Bending vibration of aliphatic $\mathrm{C}-\mathrm{H}$ \\
\hline 1026 & Bending vibration of aliphatic $\mathrm{C}-\mathrm{H}$ \\
\hline $698-756,543$ & Bending vibration of aromatic $\mathrm{C}-\mathrm{H}$ \\
\hline
\end{tabular}

TABLE 6: XRD results of Na-MMT and organo-MMT.

\begin{tabular}{lcc}
\hline Sample bentonite & $2 \theta\left(^{\circ}\right)$ & Basal spacing $(\AA)$ \\
\hline Na-MMT & 7.1 & 12.4 \\
Organo-MMT & 4.5 & 19.6 \\
\hline
\end{tabular}

absorptions bands that are attributable to PS and organoMMT. All PS bands appeared in these spectra with slight shifting. New bands that appeared in the regions of 3625 , 1027 , and $466 \mathrm{~cm}^{-1}$ were attributed to $\mathrm{OH}$ stretching of structural hydroxyl groups (Al-OH), Si-O stretching, and bending vibrations of organo-MMT and were indicating the existence of organo-MMT in the PS matrix where the polymer chain was inserted between the layers of the organoMMT by secondary valence forces.

\subsection{X-Ray Diffraction}

3.3.1. X-Ray Diffraction of Montmorillonite. XRD analysis provides information on changes in the interlayer spacing of the clay layers. The formation of an intercalated structure should result in a decrease in the $2 \theta$ value, which would indicate an increase in $d$ spacing, whereas the formation of an exfoliated structure usually results in the complete loss of registry between the clay layers such that no peak can be observed in the XRD trace [4]. Thus, the Na-MMT and organo-MMT samples were studied by WAXD measurements in the range of $2 \theta=(1-10)^{\circ}$. Figure 5 shows the XRD patterns of Na-MMT and organo-MMT. Upon intercalation of $\mathrm{CTAB}$, the basal spacing increased as expected. The XRD pattern of Na-MMT and the characteristic peak (001) reflections of montmorillonite appeared at $2 \theta=7.1^{\circ}$, which corresponds to $12.4 \AA$, and this peak in organo-MMT was observed at a lower angle compared to Na-MMT. The $d$ spacing increased from $12.4\left(2 \theta=7.1^{\circ}\right)$ for Na-MMT to $19.6 \AA\left(2 \theta=4.5^{\circ}\right)$ for organo-MMT. This increase in $d$ spacing provides evidence to support the exchange of the interlayer sodium by $\mathrm{CTA}^{+}$ions. Figure 6 demonstrates the probable mechanism of adsorbing CTAB molecules onto the montmorillonite surface layers as well as the relationship between $\mathrm{CTAB}$ adsorption and the structure of the adsorption layer influenced by the charge distribution of the clay surface. Initially, the clay saturated by a concentration of CTAB less than a CEC of 0.7, CTAB adsorption occurs by cation exchange (Figure 6(a)) [27], which causes nonuniform interlayer swelling. As the CTAB concentration approaches the CEC (0.8-1.0 CEC) of clay, the selective intercalation of CTAB develops along the silicate layers (Figure 6(b)). When the CTAB concentration increases beyond the CEC $(>1.0$ CEC) of the clay, CTAB adsorption may predominantly occur through hydrophobic bonding, which increases interlayer spacing to $\sim 40$ (Figures 6(c) and 6(d)) [27]. The result, which was obtained for organo-MMT, confirms the probable mechanism where the $d$ spacing of the (001) peak shifts directly from $12.4 \AA\left(2 \theta=7.1^{\circ}\right)$ in the Na-MMT to $19.6 \AA\left(2 \theta=4.5^{\circ}\right)$ in the organo-MMT (see Table 6). 
TABLE 7: The XRD data of organo-MMT and PS/MMT nanocomposite spectra.

\begin{tabular}{|c|c|c|c|c|c|c|c|c|c|}
\hline \multirow{2}{*}{\multicolumn{2}{|c|}{ Organo-MMT }} & \multicolumn{8}{|c|}{ PS/organo-MMT nanocomposites at different sonication time } \\
\hline & & \multicolumn{2}{|r|}{$0.5 \mathrm{~h}$} & \multicolumn{2}{|r|}{$1 \mathrm{~h}$} & \multicolumn{2}{|c|}{$1.5 \mathrm{~h}$} & \multicolumn{2}{|r|}{$2 \mathrm{~h}$} \\
\hline $2 \theta^{\circ}$ & $d$-spacing $\AA$ & $2 \theta^{\circ}$ & $d$-spacing $\AA$ & $2 \theta^{\circ}$ & $d$-spacing $\AA$ & $2 \theta^{\circ}$ & $d$-spacing $\AA$ & $2 \theta^{\circ}$ & $d$-spacing $\AA$ \\
\hline- & - & 3.4 & 25.96 & 3.4 & 25.96 & 3.6 & 25.53 & 3.4 & 25.96 \\
\hline 4.5 & 19.6 & 4.7 & 18.785 & 4.8 & 18.39 & 4.5 & 18.019 & 4.7 & 18.786 \\
\hline 6.8 & 12.988 & 6.8 & 12.988 & 7.2 & 12.267 & 7.4 & 11.936 & 7.4 & 11.936 \\
\hline 9 & 9.818 & 9.1 & 9.7099 & 8.8 & 10.04 & - & - & 7.8 & 11.3251 \\
\hline
\end{tabular}



(a) Monolayer

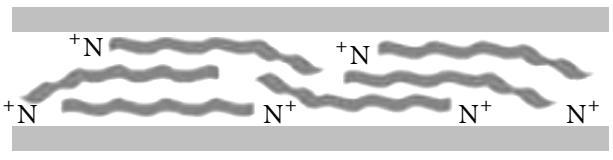

(c) Pseudotrilayer

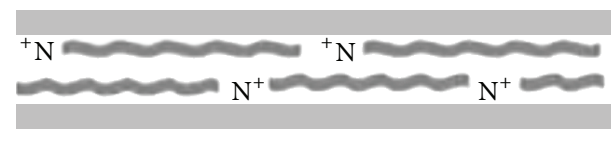

(b) Bilayer

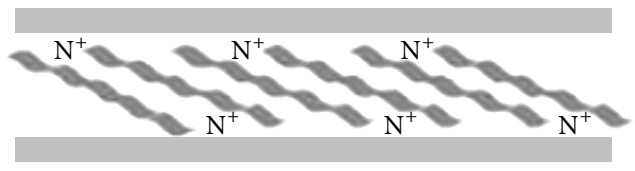

(d) Paraffin structure

Figure 6: Potential configuration of interlayer cations.

3.3.2. X-Ray Diffraction of Nanocomposites. X-ray diffraction (XRD) measurements were also applied to analyze the structural characteristics of PS and PS nanocomposites, which were prepared at different sonication times. Figure 7 shows the XRD patterns of PS and PS/organo-MMT nanocomposites, and Table 7 shows the $2 \theta$ and $d$-spacing values. For PS, only a broad amorphous peak, which appeared within the range of $2 \theta=(18-22)^{\circ}$, can be observed. For PS/organo-MMT nanocomposites, it is clear from figure that some diffraction peaks of organo-MMT and the broad peak of PS appeared in the spectra for all nanocomposite samples. The diffraction peak of montmorillonite, which appears at $2 \theta=4.5^{\circ}$ in the organo-MMT spectrum, appeared at a smaller angle with increasing $d$ spacing for all PS/MMT nanocomposite spectra, and the angle was approximately the same for all samples (see Table 7). The increase in $d$ spacing indicates that intercalated nanocomposites were formed. It was also noticed from the figure that there are sharp peaks that clearly appeared in the region of amorphous PS with increasing sonication times. This may indicate that the crystalline structure of the polymer was positively affected. An optimal sonication time of $1 \mathrm{~h}$ was found to produce nanocomposites with maximum peak intensity. The sonication time for 0.5 hour is not enough for the order and regularity of polymer chains between the interlayers of $\mathrm{MB}$. At sonication time of $1 \mathrm{~h}$ resulted in region with crystalline structure (Figure 7). When the time of sonication increases more than an hour this may be lead to entering the polymer chains between the clay layers with random orientation due to the instability of the structure of clays. However, the effect of sonication on the clay polymers nanocomposites still needs further studies to identify the regularity of polymer chains between the clay layers.

3.4. Transmission Electron Microscopy (TEM). Transmission electron microscopy (TEM) provides an actual image of the clay layers, which permits the identification of the morphology of the nanocomposites [4]. Thus, TEM was another method to observe evidence for the formation of the intercalated structure of the prepared nanocomposites. Figure 8 shows the TEM images of PS/organo-MMT at $1 \mathrm{~h}$ of sonication time at a variety of magnifications. As shown in these images, the clay platelets were still stacked on each other (see the darkest areas). In addition, the clay layers have expanded with varied interlayer spacing, such as $2.15,2.56$, and $2.6 \mathrm{~nm}$, whereas little individual clay layers were well distributed in the polymer matrix. Finally, from these images, it can be determined that intercalated structures and a very small amount of exfoliated structures were formed, which confirms and agrees with the XRD results.

\subsection{Scanning Electron Microscopy (SEM)}

3.5.1. SEM of Montmorillonite. To determine the shape, size, and morphology of the particles, SEM was used. In Figure 9, micrographs of powder of raw MMT, Na-MMT, and organoMMT samples are presented. It is clear that particles of all samples are irregularly shaped and contain many edges with different sizes. It is known that these factors play an important role in the interaction between filler, matrix, and interfacial adhesions. For the morphology of the samples, the images showed that the original Na-MMT had massive and 


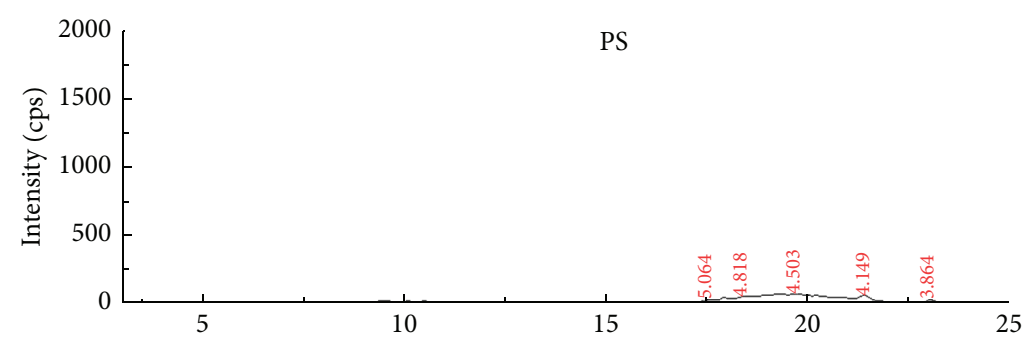

(a)

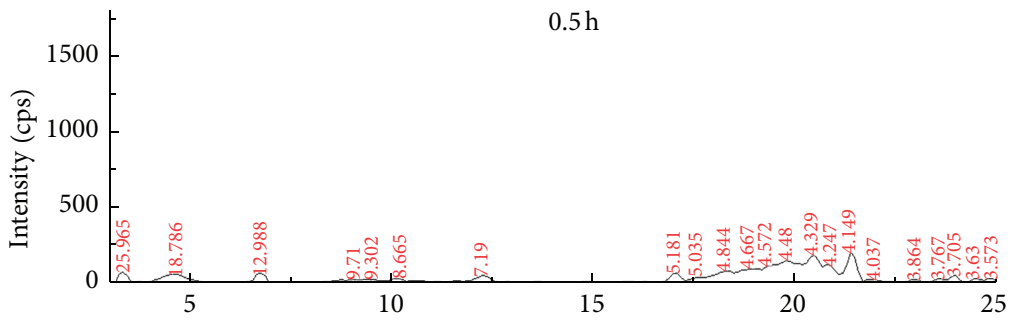

(b)

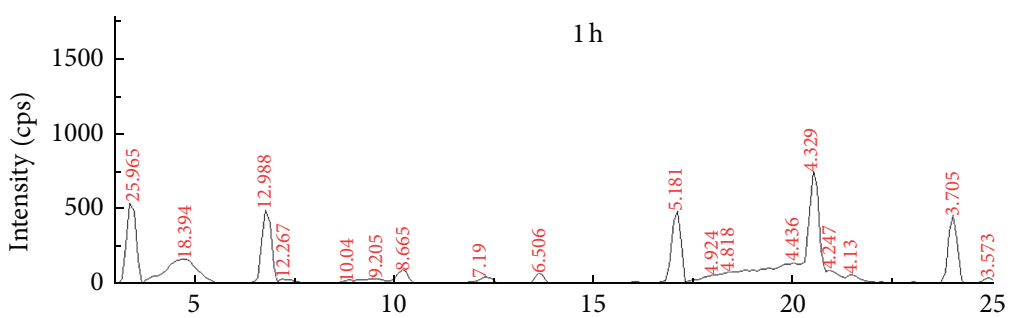

(c)

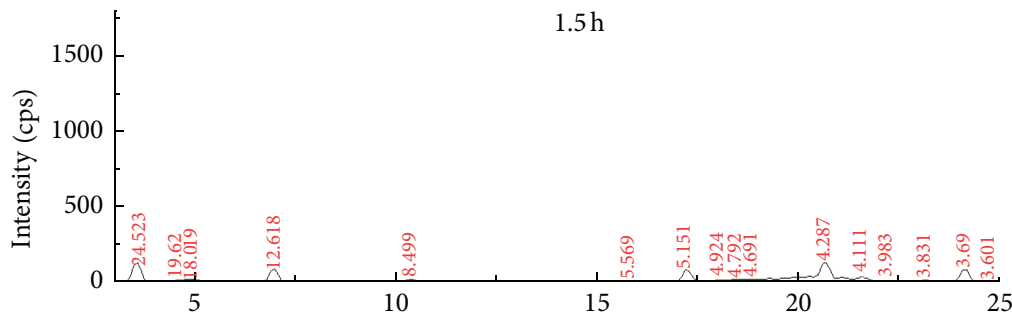

(d)

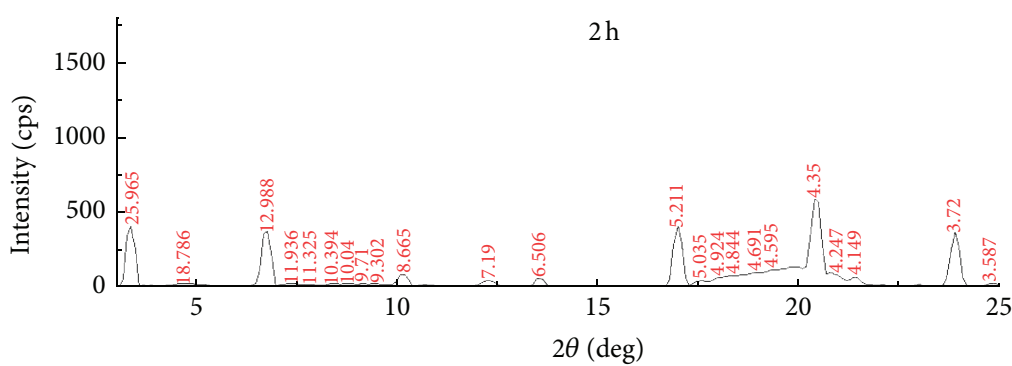

(e)

FIGURE 7: XRD patterns of organo-MMT, PS, and PS/MMT nanocomposites at different sonication times.

curved plates. Compared to the morphology of Na-MMT, the montmorillonite clay, which was modified with CTAB, showed significant changes in morphology, and there were a lot of aggregated particles, and the plates became flat.
3.5.2. SEM of Nanocomposites. To observe the dispersion of the particles, the nanocomposites after $1 \mathrm{~h}$ of sonication were examined using SEM, and the images are presented in Figure 10. Incorporation of $10 \%$ organo-MMT with polystyrene 


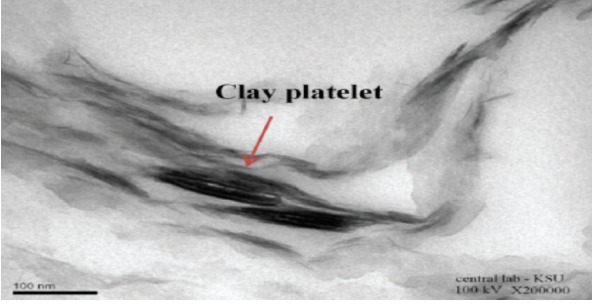

(a)

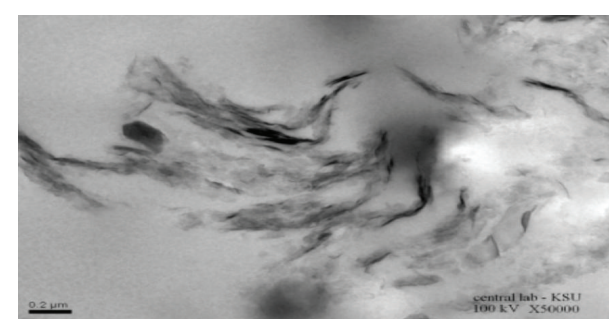

(b)

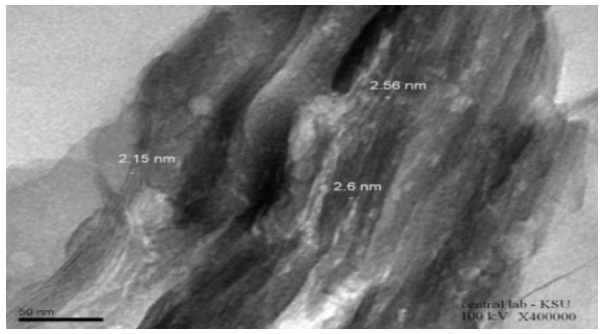

(c)

FIGURE 8: TEM images of PS/organo-MMT after $1 \mathrm{~h}$ of sonication time.

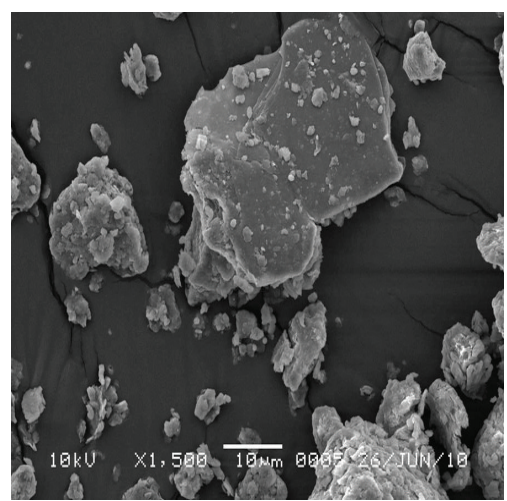

(a)

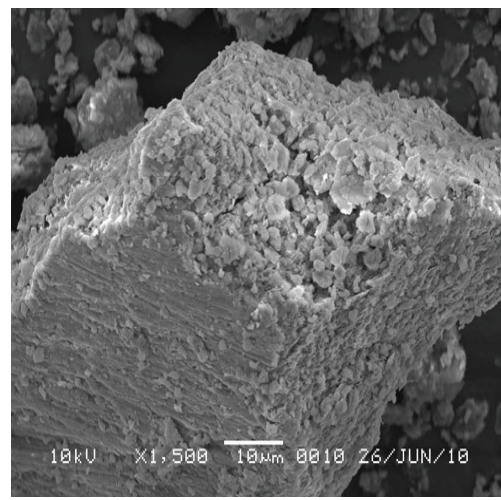

(b)

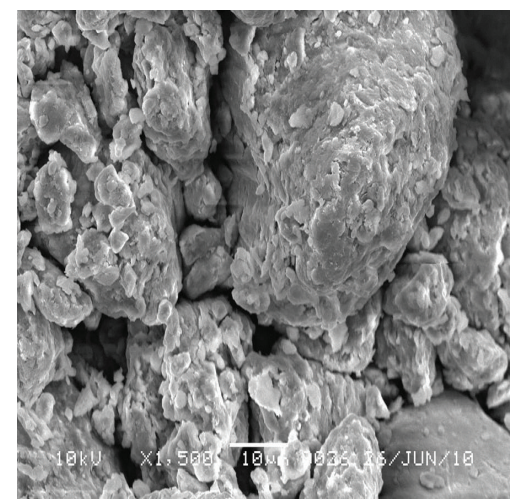

(c)

Figure 9: SEM images of raw MMT (a), Na-MMT (b) and organo-MMT (c). 


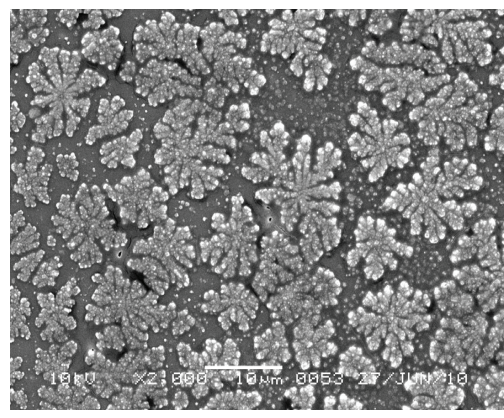

(a)

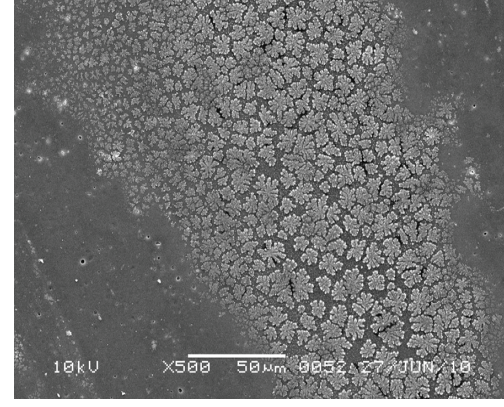

(b)

FIGURE 10: SEM images of PS/organo-MMT after $1 \mathrm{~h}$ of sonication time at two magnification.

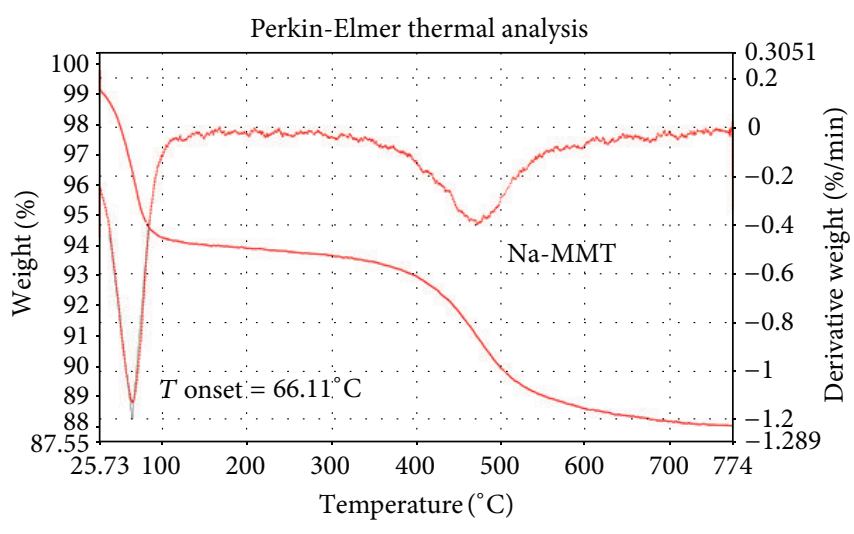

(a)

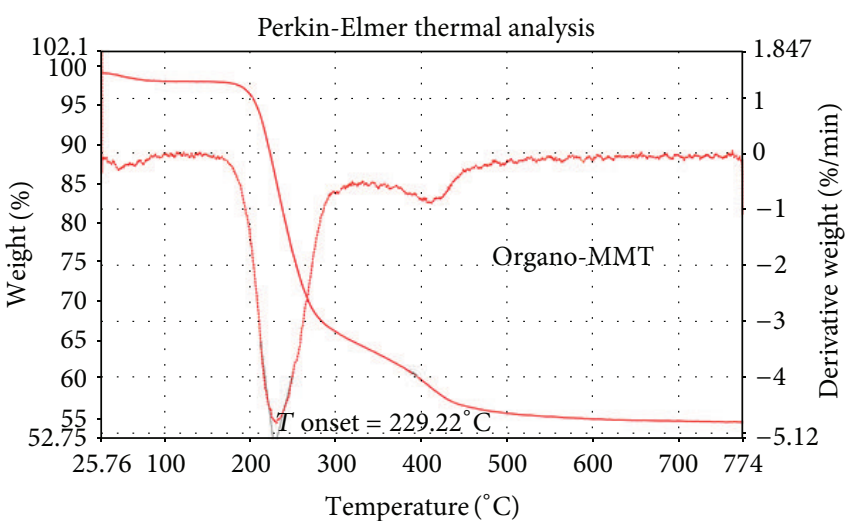

(b)

FIGURE 11: TGA curve of Na-MMT and organo-MMT.

matrix resulted in a region with spherulite texture (lamella structure). This texture could be related to the crystallinity of the polymer. Spherulite-looking crystals with beautiful impinged boundaries were observed in PS/organo-MMT after $1 \mathrm{~h}$ of sonication time compared to PS. Good dispersion of this percentage of MMT throughout the PS matrix under sonication could be led to a nucleation effect and increase the percentage of crystallinity. This result strongly agrees with the XRD results.

\subsection{Thermogravimetric Analysis (TGA)}

3.6.1. TGA of Montmorillonite. Weight loss because of the formation of volatile products after degradation at high temperature was monitored as a function of temperature. The data, which were obtained using TGA analysis, included the temperature at which $10 \%$ degradation occurred, which is a measure of the onset temperature of degradation [4]. Figure 11 shows the TG curves as well as the corresponding derivative DTG curves of Na-MMT and organo-MMT. For Na-MMT, thermal decomposition occurred in two steps. For organo-MMT, the thermal decomposition took place in three steps. The first step of the TG graph of Na-MMT up to $110^{\circ} \mathrm{C}$ was related to the dehydration of water molecules adsorbed in pores and between the silicate layers during heating, such that the first endothermic peak appeared at $T$ onset $66^{\circ} \mathrm{C}$ in the
DTG graph, which is where the mass loss accounted for $13 \%$ at $110^{\circ} \mathrm{C}$. For organo-MMT, the dehydration peak appeared smaller in the DTG graph, and the corresponding mass loss percentages was $2 \%$.

The second step of the TG curve of organo-MMT began at approximately $200-216^{\circ} \mathrm{C}$ and ended at approximately $300-350^{\circ} \mathrm{C}$, and the maximum mass loss rates occurred at $T$ onset $229^{\circ} \mathrm{C}$. At the end of this stage, the mass loss percentage of organo-MMT was $13 \%$. These losses were attributed to the thermal degradation of the alkyl tails $\left(-\mathrm{CH}_{2}\right)$ and ammonium heads $\left(-\mathrm{N}\left(\mathrm{CH}_{3}\right)_{3}\right)$ [18]. In the third step of TG, which went up to $600^{\circ} \mathrm{C}$, thermal degradations lowered down, and decomposition was attributed to the remaining alkyl chains. These findings verify the successful bonding of CTAB to the montmorillonite surface and the replacement of the $\mathrm{OH}$ groups [28-31]. The residues at 600 and $450^{\circ} \mathrm{C}$, which were roughly $12 \% \mathrm{Na}-\mathrm{MMT}$ and $45 \%$ organo-MMT, respectively, were also distinctive, which explains the permanent structural modifications of the clay, although they retained their inorganic character.

3.6.2. TGA of Nanocomposites. The thermal stability of the nanocomposites and virgin PS were studied by TGA. Figure 12 and Table 8 show the TG mass loss curves and corresponding derivative curves (DTG) of PS and a series of PS/organo-MMT nanocomposites, which were prepared at 




(a)

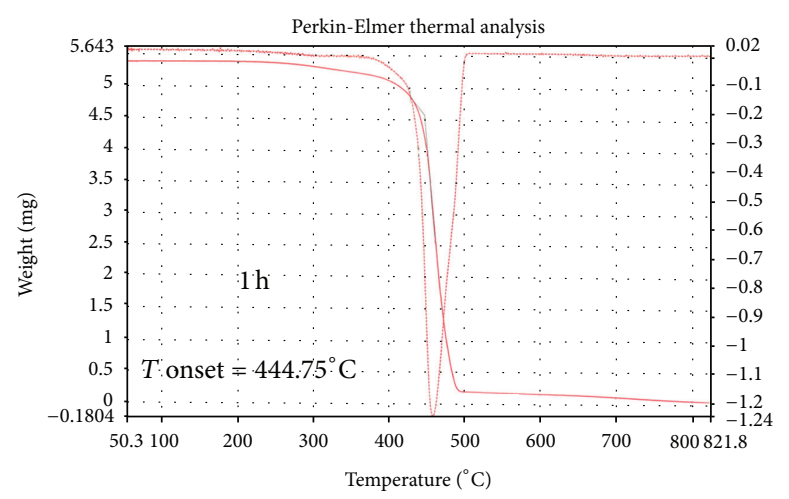

(c)

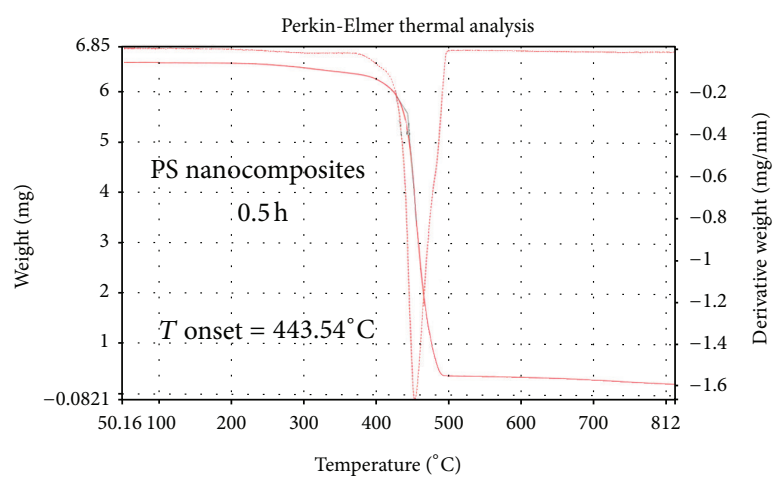

(b)

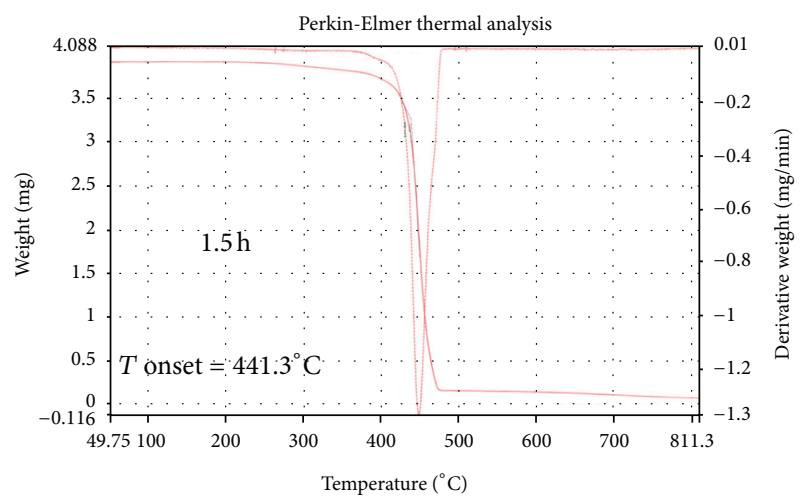

(d)

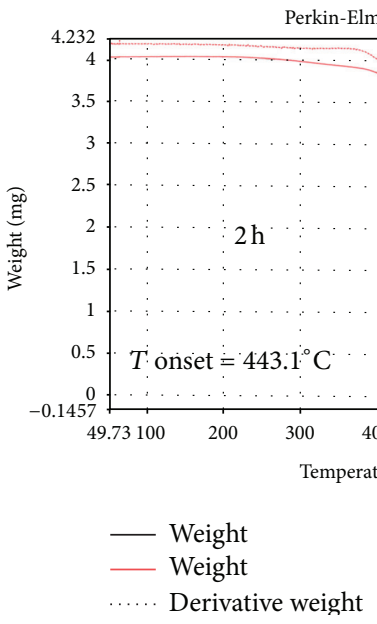

(e)

FIGURE 12: TGA curve of virgin PS and its nanocomposites at different sonication times.

TABLE 8: TG data of PS and PS/organo-MMT nanocomposites at different sonication times.

\begin{tabular}{lcccc}
\hline Samples & $T_{\text {onset }}\left({ }^{\circ} \mathrm{C}\right)$ & Weight loss $\%$ & DTG $\left(T_{\max }\right)$ & Char $\%$ \\
\hline PS & 388.07 & 100 & 430 & 0 \\
PS/10MB $2 / 0.5 \mathrm{~h}$ & 443.54 & 85.119 & 457 & 14.881 \\
PS/10MB $/ 1 \mathrm{~h}$ & 444.75 & 84.892 & 460 & 15.108 \\
PS/ $10 \mathrm{MB}_{2} / 1.5 \mathrm{~h}$ & 441.30 & 83.458 & 453 & 16.542 \\
\hline
\end{tabular}


various sonication times $(0,0.5,1,1.5$, and $2 \mathrm{~h})$. For the PS sample, the curve shows a single degradation step, which is the decomposition of the polymer backbone, and an onset temperature of $388.07^{\circ} \mathrm{C}$. The thermal decomposition of all PS/organo-MMT samples exhibit a single degradation step of weight loss from 450 to $500^{\circ} \mathrm{C}$. All samples exhibit an increase in the onset temperature of degradation relative to virgin PS, which indicates an enhancement in thermal stability. From the DTG curve, the maximum rate of change of the curve (DTG) of PS/organo-MMT nanocomposites appeared at $457,460,453$, and $457^{\circ} \mathrm{C}$ for $0.5,1,1.5$, and $2 \mathrm{~h}$ of sonication time, respectively. The improvement in thermal stability of PS/organo-MMT nanocompositesis explained as follows. First, formation of organo-MMT, which acts as a mass transport barrier and insulator between the polymer and superficial zone where polymer decomposition takes place $[32,33]$. Second, it can be attributed to restricted thermal motions of the polymer localized in the galleries [34]. Third, the increase in the crystallinity of PS, which was suggested from the XRD and SEM results. However, it is clear that there is little difference between the $T$ onset readings of the nanocomposites at different sonication times. This indicates that the difference in sonication time does not play an important role on the thermal decomposition of nanocomposites.

\section{Conclusions}

Nanocomposites containing locally produced polystyrene and modified local montmorillonite were prepared. The modification of montmorillonite was achieved using CTAB with concentrations of 2.0 CEC. Preparation of PS/organoMMT using the solution method by applying different sonication times was tested. The results of FT-IR, XRD, and SEM analyses have shown the following.

(i) The modification of the montmorillonite surface by CTAB is successfully intercalated into the clay gallery.

(ii) Using sonication during PS nanocomposite preparation gives good miscibility between organo-MMT and the PS matrix.

(iii) The type of nanocomposites of PS/organo-MMT is intercalation.

(iv) The improvement in thermal stability of PS/organoMMT nanocomposites is noted.

(v) The difference in the sonication time that was applied during preparation stage is not an important factor on the thermal stability of PS/nanocomposites.

Lastly, the study of a wide range of sonication times during the preparation stage with different materials is recommended to give a clear picture and excellent judgment regarding this factor.

\section{Acknowledgment}

The authors extend their appreciation to the Deanship of Scientific Research at King Saud University for funding the work through the research group project No. RGP-VPP-102.

\section{References}

[1] T. A. Elbokl and C. Detellier, "Aluminosilicate nanohybrid materials. Intercalation of polystyrene in kaolinite," Journal of Physics and Chemistry of Solids, vol. 67, no. 5-6, pp. 950-955, 2006.

[2] M. Alexandre and P. Dubois, "Polymer-layered silicate nanocomposites: preparation, properties and uses of a new class of materials," Materials Science and Engineering R, vol. 28, no. 1, pp. 1-63, 2000.

[3] B. K. G. Theng, The Chemistry of Clay-Organic Reactions, Wiley, New York, NY, USA, 1974.

[4] X. Zheng, D. D. Jiang, and C. A. Wilkie, "Polystyrene nanocomposites based on an oligomerically-modified clay containing maleic anhydride," Polymer Degradation and Stability, vol. 91, no. 1, pp. 108-113, 2006.

[5] A. S. Moet and A. Akelah, "Polymer-clay nanocomposites: polystyrene grafted onto montmorillonite interlayers," Materials Letters, vol. 18, no. 1-2, pp. 97-102, 1993.

[6] P. B. Messersmith and E. P. Giannelis, "Synthesis and characterization of layered silicate-epoxy nanocomposites," Chemistry of Materials, vol. 6, no. 10, pp. 1719-1725, 1994.

[7] D. C. Lee and L. W. Jang, "Preparation and characterization of PMMA-Clay hybrid composite by emulsion polymerization," Journal of Applied Polymer Science, vol. 61, no. 7, pp. 1117-1122, 1996.

[8] T. Lan, P. D. Kaviratna, and T. J. Pinnavaia, "Epoxy self-polymerization in smectite clays," Journal of Physics and Chemistry of Solids, vol. 57, no. 6-8, pp. 1005-1010, 1996.

[9] D. J. Greenland, "Adsorption of polyvinyl alcohols by montmorillonite," Journal of Colloid Science, vol. 18, no. 7, pp. 647-664, 1963.

[10] E. Ruiz-Hitzky, "Conducting polymers intercalated in layered solids," Advanced Materials, vol. 5, no. 5, pp. 334-340, 1993.

[11] H. G. Jeon, H. T. Jung, S. W. Lee, and S. D. Hudson, "Morphology of polymer/silicate nanocomposites: high density polyethylene and a nitrile copolymer," Polymer Bulletin, vol. 41, no. 1, pp. 107-113, 1998.

[12] S. Wang, C. Long, X. Wang, Q. Li, and Z. Qi, "Synthesis and properties of silicone rubber/organomontmorillonite hybrid nanocomposites," Journal of Applied Polymer Science, vol. 69, no. 8, pp. 1557-1561, 1998.

[13] Y. Li and H. Ishida, "Solution intercalation of polystyrene and the comparison with poly(ethyl methacrylate)," Polymer, vol. 44, no. 21, pp. 6571-6577, 2003.

[14] H. Li, Y. Yu, and Y. Yang, "Synthesis of exfoliated polystyrene/montmorillonite nanocomposite by emulsion polymerization using a zwitterion as the clay modifier," European Polymer Journal, vol. 41, no. 9, pp. 2016-2022, 2005.

[15] J. Russell and A. Fraser, "Infrared methods in clay mineralogy," in Spectroscopic and Chemical Determinative Methods, pp. 11-67, 1994.

[16] J. Madejová and P. Komadel, "Baseline studies of the clay minerals society source clays: infrared methods," Clays and Clay Minerals, vol. 49, no. 5, pp. 410-432, 2001.

[17] J. Madejová, M. Janek, P. Komadel, H. J. Herbert, and H. C. Moog, "FTIR analyses of water in MX-80 bentonite compacted from high salinary salt solution systems," Applied Clay Science, vol. 20, no. 6, pp. 255-271, 2002.

[18] J. M. Cervantes-Uc, J. V. Cauich-Rodríguez, H. Vázquez-Torres, L. F. Garfias-Mesías, and D. R. Paul, "Thermal degradation 
of commercially available organoclays studied by TGA-FTIR," Thermochimica Acta, vol. 457, pp. 92-102, 2007.

[19] M. Önal and Y. Sarikaya, "Some physicochemical properties of methylammonium and ethylenediammonium smectites," Colloids and Surfaces A, vol. 312, no. 1, pp. 56-61, 2008.

[20] S. Tzavalas and V. G. Gregoriou, "Infrared spectroscopy as a tool to monitor the extent of intercalation and exfoliation in polymer clay nanocomposites," Vibrational Spectroscopy, vol. 51, no. 1, pp. 39-43, 2009.

[21] Y. Ma, J. Zhu, H. He, P. Yuan, W. Shen, and D. Liu, "Infrared investigation of organo-montmorillonites prepared from different surfactants," Spectrochimica Acta A, vol. 76, no. 2, pp. 122-129, 2010.

[22] S. Akyuz and T. Akyuz, "FT-IR spectroscopic investigations of adsorption of 2-, 3- and 4-pyridinecarboxamide on montmorillonite and saponite from Anatolia," Vibrational Spectroscopy, vol. 42, no. 2, pp. 387-391, 2006.

[23] W. Xue, H. He, J. Zhu, and P. Yuan, "FTIR investigation of CTAB-Al-montmorillonite complexes," Spectrochim Acta A, vol. 67, no. 3-4, pp. 1030-1036, 2007.

[24] K. C. Cole, "Use of infrared spectroscopy to characterize clay intercalation and exfoliation in polymer nanocomposites," Macromolecules, vol. 41, no. 3, pp. 834-843, 2008.

[25] M. Önal and Y. Sarikaya, "The effect of organic cation content on the interlayer spacing, surface area and porosity of methyltributylammonium-smectite," Colloids and Surfaces A, vol. 317, no. 1-3, pp. 323-327, 2008.

[26] Z. Li, W. T. Jiang, and H. Hong, "An FTIR investigation of hexadecyltrimethylammonium intercalation into rectorite," Spectrochimica Acta A, vol. 71, no. 4, pp. 1525-1534, 2008.

[27] S. Y. Lee and S. J. Kim, "Expansion of smectite by hexadecyltrimethylammonium," Clays and Clay Minerals, vol. 50, no. 4, pp. 435-445, 2002.

[28] G. Edwards, P. Halley, G. Kerven, and D. Martin, "Thermal stability analysis of organo-silicates, using solid phase microextraction techniques," Thermochimica Acta, vol. 429, no. 1, pp. 13-18, 2005.

[29] Y. Xi, Z. Ding, H. He, and R. L. Frost, "Structure of organoclays-an X-ray diffraction and thermogravimetric analysis study," Journal of Colloid and Interface Science, vol. 277, no. 1, pp. 116-120, 2004.

[30] W. Xie, Z. Gao, K. Liu et al., "Thermal characterization of organically modified montmorillonite," Thermochimica Acta, vol. 367-368, pp. 339-350, 2001.

[31] F. Wolters and K. Emmerich, "Thermal reactions of smectitesRelation of dehydroxylation temperature to octahedral structure," Thermochimica Acta, vol. 462, no. 1-2, pp. 80-88, 2007.

[32] W. A. Zhang, D. Z. Chen, H. Y. Xu, X. F. Shen, and Y. E. Fang, "Influence of four different types of organophilic clay on the morphology and thermal properties of polystyrene/clay nanocomposites prepared by using the $\gamma$-ray irradiation technique," European Polymer Journal, vol. 39, no. 12, pp. 2323-2328, 2003.

[33] J. Wang, J. Du, J. Zhu, and C. A. Wilkie, "An XPS study of the thermal degradation and flame retardant mechanism of polystyrene-clay nanocomposites," Polymer Degradation and Stability, vol. 77, no. 2, pp. 249-252, 2002.

[34] A. Blumstein, "Polymerization of adsorbed monolayers. I. Preparation of the clay-polymer complex," Journal of Polymer Science A, vol. 3, pp. 2665-2672, 1965. 

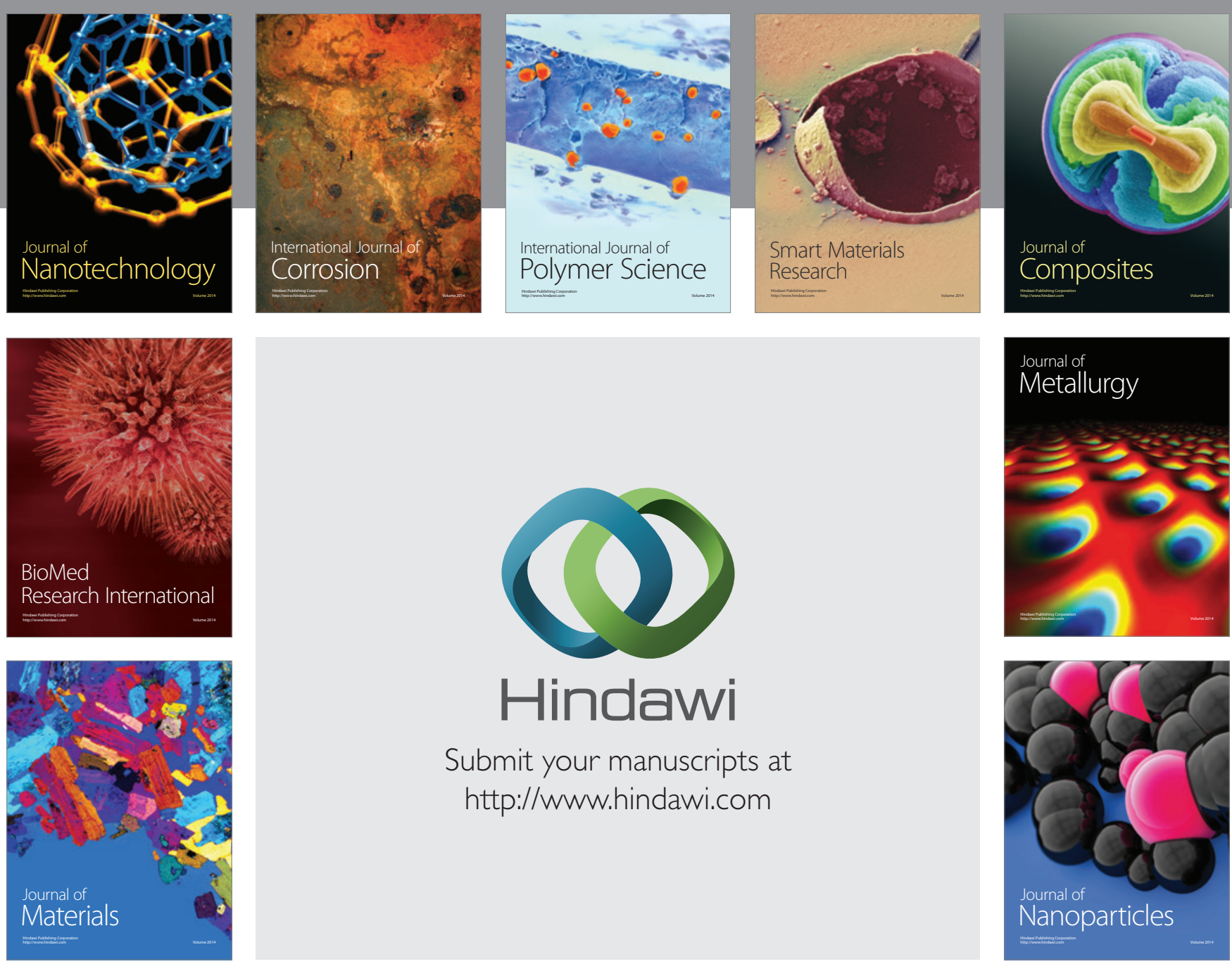

Submit your manuscripts at http://www.hindawi.com
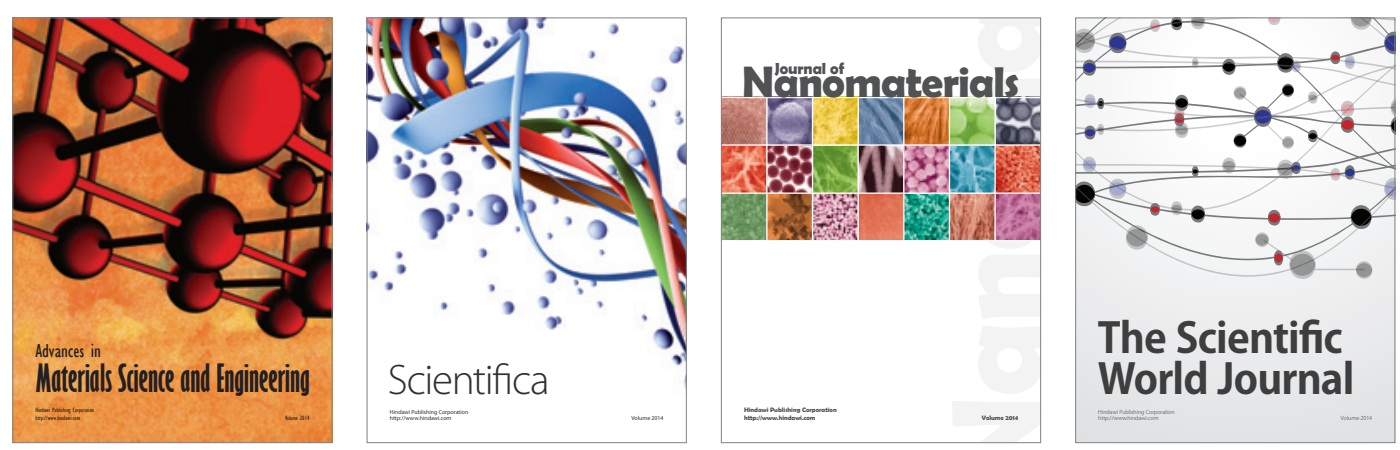

\section{The Scientific World Journal}
